Meta

Journal des traducteurs

Translators' Journal

\title{
On the Origin of Species de Charles Darwin - les métaphores et plus encore
}

\section{Eve-Marie Gendron-Pontbriand et Sylvie Vandaele}

Volume 60, numéro 2, août 2015

$60^{\mathrm{e}}$ anniversaire. Les horizons de la traduction : retour vers le futur $60^{\text {th }}$ Anniversary. Translation's Horizons: Back to the Future

60mo aniversario. Los horizontes de la traducción: regreso al futuro

URI : https://id.erudit.org/iderudit/1032887ar

DOI : https://doi.org/10.7202/1032887ar

Aller au sommaire du numéro

Éditeur(s)

Les Presses de l’Université de Montréal

ISSN

0026-0452 (imprimé)

1492-1421 (numérique)

Découvrir la revue

Citer ce document

Gendron-Pontbriand, E.-M. \& Vandaele, S. (2015). On the Origin of Species de Charles Darwin - les métaphores et plus encore. Meta, 60(2), 336-336.

https://doi.org/10.7202/1032887ar d'utilisation que vous pouvez consulter en ligne. 


\title{
On the Origin of Species de Charles Darwin - les métaphores et plus encore
}

\author{
Eve-Marie Gendron-Pontbriand et Sylvie Vandaele \\ Université de Montréal, Montréal, Canada \\ eve-marie.gendron-pontbriand@umontreal.ca; sylvie.vandaele@umontreal.ca
}

Éminent naturaliste du XIX ${ }^{\mathrm{e}}$ siècle, Charles Darwin publie en 1859 ce qui s'avérera être le texte fondateur des sciences de la vie: On the origin of species by means of natural selection, or the preservation of favoured races in the struggle for life. Cet ouvrage, que nous appellerons par commodité l'OS, pose les assises conceptuelles de sa théorie de l'évolution (TE). La TE darwinienne suscite encore de nos jours la controverse: certains la nient (créationnisme, dessein intelligent, etc.) et d'autres la poussent à l'extrême (eugénisme, darwinisme social, etc.). Vu la grande portée de l'OS, le problème de sa traduction en français se présente de lui-même. Ce champ d'étude reste pourtant largement inexploré. Seules Conry (1974), Miles (1988/1989) et Brisset (2002) s'y sont attardées, et toutes se sont penchées sur la controversée Clémence Royer, première traductrice de l'OS. Nous nous proposons donc d'étudier cinq des sept éditions anglaises de l'OS (1859, $1861,1869,1872$ et 1876) et leurs sept traductions françaises, cinq contemporaines et deux modernes. Nous nous limiterons au quatrième chapitre, qui porte sur le concept clé de la TE: la sélection naturelle. Notre recherche se situe dans la lignée des travaux de Vandaele, eux-mêmes inspirés du cadre théorique de la sémantique cognitive, notamment les travaux de Lakoff et Johnson (1980/2003), et de Talmy (2000). Nous examinerons donc les métaphores conceptuelles employées par Darwin, dont Ruse va même jusqu'à dire qu'elles confèrent son pouvoir heuristique et sa valeur épistémique à la TE, bien qu'elles soient parfois également source de confusion (Ruse, 2000/2005/2010). Pour ce faire, nous employons une méthode déjà définie par Vandaele et Boudreau (2006). Cette méthodologie nous permettra de comprendre comment les notions propres à la TE sont conceptualisées métaphoriquement et de comparer les différentes traductions françaises de l'OS. Les résultats de notre recherche jettent une lueur inédite sur la traduction d'une des œuvres scientifiques les plus marquantes des derniers siècles et contribuent aux connaissances sur l'histoire de la traduction.

Eve-Marie Gendron-Pontbriand détient un baccalauréat en microbiologie et immunologie (mention Honours) de l'Université McGill, avec mineure en langue et traduction française. Après quelques années de recherche dans le domaine biomédical, elle s'est réorientée vers le domaine de la traduction, dans le but d'étudier spécifiquement la traduction scientifique. Après avoir obtenu son DÉSS en traduction en 2011, elle a poursuivi des études à la maîtrise en traduction, qu'elle a terminées en août 2013. Elle est actuellement dans sa deuxième année de doctorat en traduction. 